\title{
AVALIAÇÃO DA POTENCIALIDADE DE FUNÇÕES OBJETIVO UTILIZADAS NA IDENTIFICAÇÃO DE FALHAS EM ESTRUTRAS TRELIÇADAS
}

\section{Objective Functions Capability Assessment Used In Identification in Damage in Truss Structures}

\author{
Marcus Alexandre Noronha de Brito ${ }^{1}$, Luciano Mendes Bezerra ${ }^{2}$, \\ William Taylor Matias Silva ${ }^{3}$, Wellington Andrade da Silva ${ }^{4}$
}

Recebido em 05 de novembro de 2016; recebido para revisão em 03 de março de 2017; aceito em 24 de abril de 2017; disponivel on-line em 16 de maio de 2017.

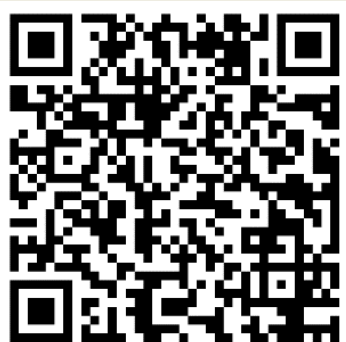

PALAVRAS CHAVE:

Funções objetivo;

Danos estruturais;

Identificação de falhas;

Treliças;

Métodos mínimos

quadrados.

\section{KEYWORDS:}

Objective functions; Structural damage; Damage identification;

Trusses;

Least square method.

* Contato com os autores:

e-mail: marcusanb@yahoo.com.br (M. A. N. Brito)

Eng. Civil, Doutorando, Professor Adjunto, Instituto Federal de Educação, Ciência e Tecnologia de Brasília.

2e-mail: Imbz@unb.br ( L. M. Bezerra)

Professor Associado, Universidade de Brasília, Programa de Pós-Graduação em Estruturas e Construção Civil, Faculdade de Tecnologia, Campus Darcy Ribeiro.

${ }^{3}$ e-mail: taylor@unb.br (W.T.M. Silva )

Professor Associado, Universidade de Brasília, Programa de Pós-Graduação em Estruturas e Construção Civil, Faculdade de Tecnologia, Campus Darcy Ribeiro.

${ }^{4}$ e-mail: wellington_andrade@ufg.br (W. A. Silva )

Professor Adjunto, Universidade Federal de Goiás, Programa de Pós-Graduação em Modelagem e Otimização - IMTec, Faculdade de Engenharia - Regional Catalão. 


\section{INTRODUÇÃO}

Diversas técnicas são atualmente utilizadas para identificação de danos em estruturas. A inspeção visual é uma das técnicas mais comumente empregada, contudo, carece da experiência e acuidade visual de quem a realiza, entretanto, existe nesta a problemática de ser incapaz de identificar danos superficiais (Brito, 2008). Já a utilização de outras técnicas como o ultrassom e o raio- $X$ constituem efetivas alternativas para se detectar a existência de danos internos à estrutura, porém, são de aplicação localizada exigindo um conhecimento prévio da região afetada, além de condições propícias de acessibilidade às áreas a serem examinadas visto o volume da aparelhagem empregada, mesmo quando portáteis (Halmshaw, 1987; Garnier, 2011).

A utilização do líquido penetrante é outro método bastante eficiente quando se trata de danos externos à estrutura, mas não apresenta resultados satisfatórios quando tais danos se localizam sob a superfície do objeto. As partículas magnéticas e a utilização da corrente de Eddy, apesar de serem métodos de investigação mais eficazes, são economicamente pouco viáveis quando aplicados em estruturas de grandes vãos, como treliças de pontes metálicas, por exemplo.

Vários outros métodos não destrutivos são aplicados e pesquisados ao longo do tempo na solução do problema da identificação de danos e acompanhamento da integridade estrutural, por exemplo com a implantação de sensores piezelétricos (Oliveira, 2013).

Com o objetivo de se encontrar as regiões danificadas muitos pesquisadores, tentam estabelecer análises prévias da estrutura com o emprego de métodos numéricos associados a técnicas de análise inversa (Tanaka e Masuda, 1986; Kubo, 1988; Bezerra e Saigal, 1993; Wang et al., 2001; Tomaszewska, 2010; Bucher, 2011). Estas técnicas de análise inversa ou Problemas Inversos que se caracterizam na identificação das causas por meio da identificação de suas consequências ou ainda que possuam muitas vezes valores de domínio do problema insuficiente ou até mesmo de forma redundante, também chamados de problemas mal postulados, problemas que muitas vezes possuem soluções não únicas (Wen, 2011), diferem substancialmente das análises diretas ou Problemas Diretos ou de Valor de Contorno, ou seja, os que possuem valores ou propriedades físicas e matemáticas bem definidas, obtendo-se classicamente as consequências em função de suas causas comumente feitas nos escritórios de Engenharia.

\section{A IDENFITICAÇÃO DE DANOS EM ESTRUTURAS}

O problema de identificação de danos em estruturas treliçadas tem merecido basicamente duas vertentes: a elastostática e a elastodinâmica. A preferência das formulações tem sido pela elastodinâmica via análises nas alterações das frequências e modos de vibrar da estrutura, comparando assinaturas da mesma estrutura obtidas antes e depois da estrutura apresentar defeito.

Segundo Brito, (2008), apesar desta atenção mais voltada para a dinâmica, nota-se que muitos problemas ainda persistem nestas formulações entre eles: (1) os modelos dinâmicos adotam formulações numéricas que não simulam as verdadeiras características dinâmicas das estruturas de uso na prática; (2) nos testes de análise modal, por vezes é difícil a obtenção dos modos de vibrar de grande parte das estruturas civis que se apresentam, geralmente, de forma muito rígida; (3) em alguns métodos usando análise modal faz-se necessária a determinação de muitos modos de vibrar, inclusive modos de alta ordem. A determinação de danos usando análise estática é mais simples e fácil de executar na prática. Este trabalho tenta unificar as duas vertentes de resolver o problema usando dados de análises estáticas bem como análises no domínio da frequência.

$\mathrm{Na}$ identificação de defeitos, o domínio da estrutura não é conhecido; i.e., as falhas (ou os danos) não são conhecidas em termos de localização e extensão. Geralmente para identificar um dano é adotado um algoritmo matemático que 
exige uma assinatura ou caracterização da estrutura e dados experimentais (Brito, 2008).

A partir de um modelo de dano idealizado (posição e forma expressas por meio de variáveis), comparam-se os valores de referência com a resposta calculada através de uma assinatura escolhida. A diferença entre modelo idealizado e a realidade gera um resíduo que depende da assinatura escolhida para caracterizar o defeito. A Figura 1 mostra o mapeamento entre um modelo de dano idealizado e o dano real nas estruturas treliçadas (Brito, 2008).

A aplicação da "Função Objetivo" ou "Assinatura Estrutural", equações que organizam matematicamente em sua formatação as características de resposta estática ou dinâmica, tem como objetivo reduzir as diferenças dos resultados obtidos entre o modelo idealizado e de referência, minimizando-se assim as "Funções Resíduo" obtém-se a melhor solução do problema, ou seja, identifica-se o dano interno da estrutura.

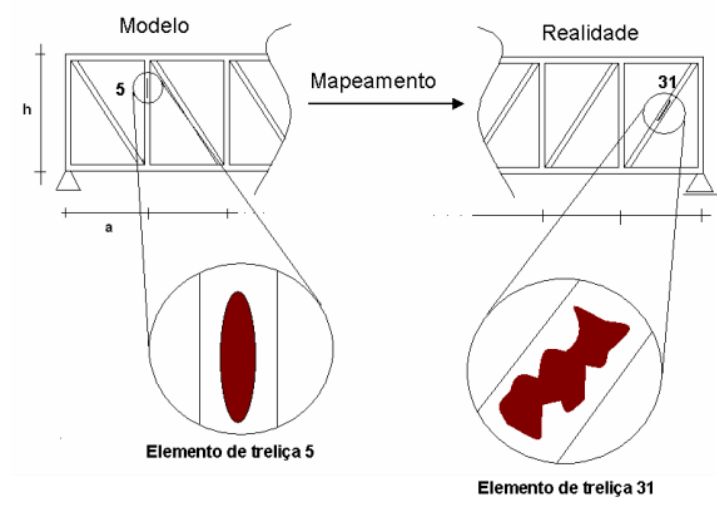

FIGURA 1: Esquema de mapeamento da estrutura - 0 modelo de referência e o modelo idealizado. FONTE: Próprios autores.

Segundo Bezerra, (1993), diversas podem ser as técnicas utilizadas para resolver os Problemas Inversos de identificação de danos em estruturas. Dentre estas técnicas, destacam-se, pela simplicidade, os métodos de "best-fit" (Figura 2) ou ajustamento da resposta prognosticada (ou calculada) a um conjunto de medidas disponíveis (ou dados de referência) com a utilização do Método dos Mínimos Quadrados - MMQ.

Nesta metodologia os danos estruturais são mapeados por meio de uma equação do tipo
$A z=\Delta \tilde{u}$ e com a utilização de técnicas de minimização, neste caso o Método dos Mínimos Quadrados. Dessa forma, as Funções Objetivo são definidas em termos de parâmetros reunidos em um vetor $z$ e variáveis que definem o modelo numérico que descreve os danos e espaços métricos apropriados. Os resultados obtidos pelo operador matemático $A$ sobre o vetor z é comparado a $\Delta \tilde{u}$ que é um vetor de dados de referência, buscandose assim a minimização do processo, e em conseguinte, obtenção da melhor solução.

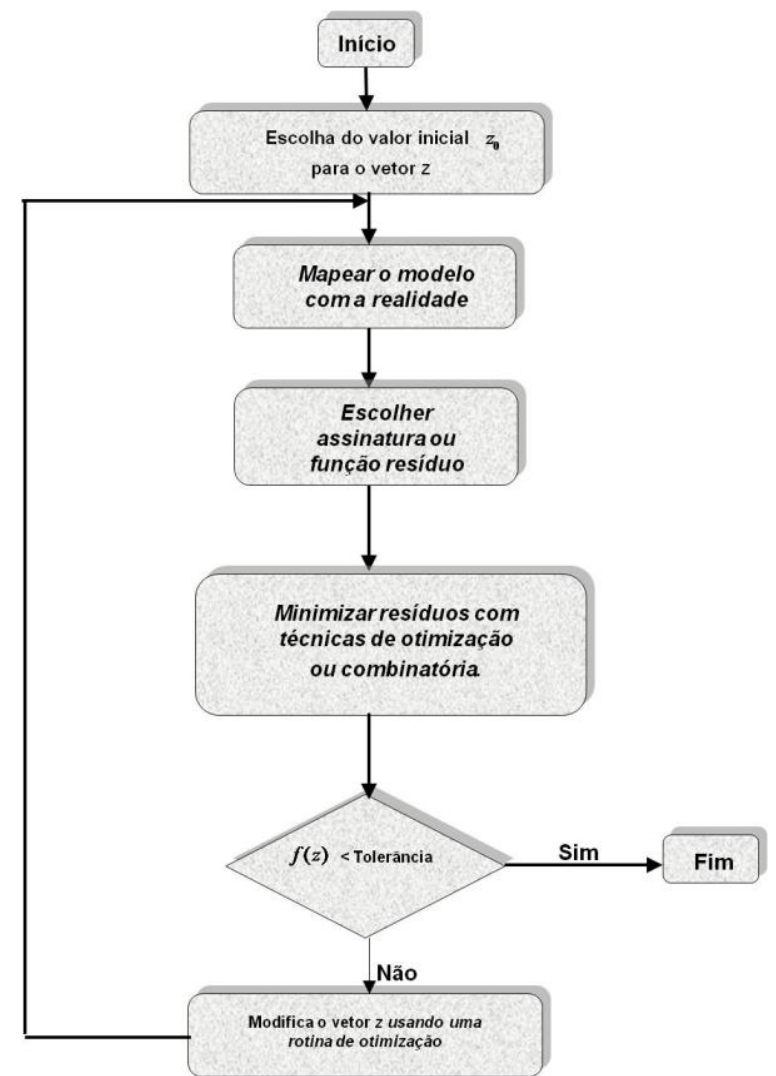

FIGURA 2: Esquema do algoritmo de otimização entre o modelo de referência e o modelo idealizado da estrutura.

FONTE: Próprios autores.

Os dados de referência são valores que revelam a diferença de comportamento característico da estrutura. Na relação matemática $A z=\Delta \tilde{u}$, " $A$ " é um operador matemático sobre as variáveis $z$ ajustadas aos dados de referência $\Delta \tilde{u}$. Assim, a metodologia parte de valores iniciais de $z$, onde o método faz com que as variáveis no vetor $z$ sejam tais que o mapeamento $A z$ reproduza o conjunto de dados experimentais $\Delta \tilde{u}$.

Na evolução do vetor $z$, a partir de valores 
iniciais, a diferença entre $A z$ e $\Delta \tilde{u}$ é definida como um resíduo que será representado por uma função que depende da assinatura escolhida. A função resíduo deve ser definida por uma norma num espaço métrico apropriado (Scales e Gersztenkorn, 1988).

As falhas (ou os danos) não são conhecidas em termos de localização e de extensão, entretanto, devem ser encontradas a partir do vetor de dados $\Delta \tilde{u}$. É importante notar que $\Delta \tilde{u}$ deve levar em conta alguma diferença de característica comportamental da estrutura, entre elas destacam-se as respostas em deslocamento, frequências, deformações, tensões, reações de apoio, etc. Nota-se que o conhecimento de técnicas de medição experimental de deslocamentos e frequências são comuns e que a maioria dos autores que trabalha com técnicas inversas de identificação de falhas em estruturas adotam valores de referência $\Delta \tilde{u}$ advindos de deslocamentos ou frequências ou raramente combinação entre eles (Kubo, 1988; Tanaka e Masuda, 1986; Tanaka et al. 1988; Bezerra, 1993; Hu et al., 2001), .

Neste trabalho é feita uma análise inicialmente modal para a determinação das frequências e modos de vibrar da estrutura e subsequentemente diversos carregamentos estáticos nodais são aplicados na estrutura intacta e com defeito a fim de se determinar o vetor $\Delta \tilde{u}$.

\section{O COMPORTAMENTO DA RIGIDEZ, DESLOCAMENTO E FREQUÊNCIAS NATURAIS}

Em uma estrutura sem defeito, o modelo analítico para calcular a resposta da estrutura sob carga estática $p$ pode ser expresso pela Equação 1.

$$
\mathrm{Ku}=\mathrm{p},
$$

Em que:

$\mathrm{K}=$ matriz de rigidez da estrutura e u é o vetor de deslocamento da estrutura.

Uma estrutura ao apresentar um dano, sofre uma variação em sua rigidez expressa matematicamente por uma variação $\Delta \mathrm{K}$ na matriz de rigidez $\mathrm{K}$. Um dano pode também causar perda de massa e alteração nas características de amortecimento da estrutura, entretanto, estas variações são desprezíveis como considerações deste trabalho.

Portanto, a estrutura danificada sob a nova equação de equilíbrio é expressa conforme Equação 2:

$$
(\mathrm{K}+\Delta \mathrm{K}) \mathrm{u}^{\wedge *}=\mathrm{p} \quad \text { Eq. [2] }
$$

Em que:

$\mathrm{u}^{*}=$ vetor de deslocamento da estrutura com dano. Considerando uma aproximação de primeira ordem, $\mathrm{u}^{*}$ é denotado por meio da Equação 3:

$$
\begin{array}{r}
\mathrm{u}^{*}=(\mathrm{K}+\Delta \mathrm{K})^{-1} \mathrm{p} \approx\left(\mathrm{K}^{-1}-\mathrm{K}^{-1} \Delta \mathrm{KK}^{-1}\right) \mathrm{p} . \\
\text { Eq. [3] }
\end{array}
$$

A variação $\Delta u$ entre estrutura com e sem dano pode ser calculada pela Equação 4:

$$
\Delta \mathrm{u}=\mathrm{u}-\mathrm{u}^{*}=\mathrm{K}^{-1} \Delta \mathrm{KK}^{-1} \mathrm{p} \Rightarrow \mathrm{K}(\Delta \mathrm{u})=\underset{\text { Eq. [4] }}{(\Delta \mathrm{K}) \mathrm{u}}
$$

Do ponto de vista do comportamento em vibração livre da estrutura, a análise modal revela as frequências naturais através da resolução da Equação 5 de autopares.

$$
\mathrm{K} \Phi=\omega^{2} \mathrm{M} \Phi
$$

Em que:

$\mathrm{K}=$ Matriz de rigidez da estrutura sem dano;

$\mathrm{M}=$ Matriz de massa da estrutura sem dano;

$\omega_{\mathrm{j}}=$ Autovalores do sistema;

$\Phi_{\mathrm{j}}=$ Autovetores do sistema.

A indicação do quadrado da frequência, $\Delta \omega_{\mathrm{j}}^{2}$, pela presença do dano na estrutura é obtida por uma aproximação de primeira ordem, dada pela Equação 6:

$$
\Delta \omega_{j}^{2} \approx \frac{\Phi_{j}^{T} \Delta K \Phi_{j}}{\Phi_{j}^{T} M \Phi_{j}}
$$

Observa-se, no entanto, que na equação precedente a variação da frequência não leva em 
conta a perda (variação) de massa $\Delta \mathrm{M}$. Isso limita a aplicabilidade da Equação 6 ao cálculo da variação de frequências para estruturas cujos danos impliquem em perdas significativa de massa, como por exemplo, estruturas sob corrosão.

As Equações 4 e 6 justificam as variações de deslocamento e frequências em estruturas apenas com falhas devido à perda de rigidez. Observa-se que, devido ao aparecimento de uma falha, as variações de deslocamento e de frequência mostram-se sensíveis à variação $\Delta \mathrm{K}$, e, portanto, consideradas em assinaturas estruturais avaliadas.

A seguir são definidas algumas "Assinaturas Estruturais" ou Funções Objetivo para se identificar a localização de uma falha ou dano em funções das variações de seus deslocamentos $\Delta \mathrm{u} e$ de suas frequências naturais $\Delta \omega^{2}$ e, consequentemente, dos autovalores associados $\Delta \varphi$.

\section{DEFINIÇÃO DAS FUNÇÕES OBJETIVO}

Cinco "Assinaturas Estruturais" ou Funções Objetivo com características estáticas e dinâmicas são apresentadas com possibilidade de aplicação em qualquer geometria estrutural formada com elementos de barra.

A primeira assinatura estrutural analisada, aqui denominada de $F_{1}(z)$, é uma proposição de combinação entre grandezas estáticas e dinâmicas e é descrita na Equação 7.

$\mathrm{O}$ termo $\Delta \mathrm{u}$ corresponde à diferença entre os deslocamentos nodais da estrutura intacta $u^{\mathrm{i}} \mathrm{e}$ da estrutura danificada $\mathrm{u}^{\mathrm{d}}$ nas direções $\mathrm{x}$ e y para os $\mathrm{N}$ nós da estrutura. $\Delta \omega_{1}^{2}$ e $\Delta \omega_{2}^{2}$ são os quadrados das diferenças entre as freqüências naturais $\omega$ obtidas com a estrutura intacta $\omega^{\mathrm{i}}$ e com a estrutura danificada $\omega^{\mathrm{d}}$, somente considerando a primeira e a segunda frequência natural de vibração da estrutura. Nesta assinatura, Equação 7, há um somatório de coeficientes entre deslocamentos e frequências naturais da estrutura computada para todos os nós da treliça. As variações de deslocamento e de frequências naturais são apresentadas nas Equações 8 a 10.

A segunda assinatura proposta, $F_{2}(z)$, descrita na Equação 11 apresenta apenas grandezas dinâmicas como autovalores (frequências naturais) e autovetores (modos de vibração). Esta equação considera a razão entre os quadrados das diferenças dos respectivos modos de vibração (autovetores) normalizados $\Delta \varphi^{2}$ e das frequências naturais (autovalores) $\Delta \omega^{2}$ da estrutura intacta e da estrutura danificada. Nesta assinatura, ao contrário da primeira, são usadas $\mathrm{n}_{\mathrm{f}}$ frequências naturais.

A assinatura seguinte, $F_{3}(z)$, apresentada na Equação 13 incorpora apenas valores dos modos de vibração normalizados da estrutura, denominada COMAC (Coordinate Modal Assurance Criterion). Aqui também são usados todos os $\mathrm{N}=\mathrm{n}_{\mathrm{f}}$ modos de vibrar computados para a estrutura.

A próxima assinatura é uma variação da equação COMAC apresentada anteriormente. Nesta assinatura, há uma substituição dos autovetores pelos autovalores ou frequências naturais de vibração da estrutura nas $\mathrm{N}=\mathrm{n}_{\mathrm{f}}$ frequências extraídas, resultando na assinatura $\mathrm{F}_{4}(\mathrm{z})$, mostrada na Equação 14.

Com a assinatura proposta $\mathrm{F}_{4}(\mathrm{z})$ se possibilita uma análise comparativa em relação à assinatura $F_{3}(z)$ descrita na Equação 13 que utiliza os modos de vibração da estrutura em vez das frequências naturais obtidas.

A assinatura $F_{5}(z)$ dada pela equação seguinte tem formulação estático-dinâmica. Assim como a função $F_{1}(z)$, apresentada na Equação 7, esta nova assinatura utiliza diferenças de deslocamentos estáticos nas duas direções $x$ e $y\left(\Delta u_{x}^{j}+\Delta u_{y}^{j}\right)$ e diferenças do quadrado das frequências naturais, $\Delta \omega_{\mathrm{j}}^{2}$, entre a estrutura intacta e a estrutura danificada. A assinatura é analisada para todos os $\mathrm{N}$ graus de liberdade das barras da treliça e para as $\mathrm{K}$ primeiras frequências naturais extraídas para a estrutura.

$$
F_{1}(z)=\sum_{j=1}^{N}\left[\left(\frac{\Delta u_{x}^{j}}{\Delta \omega_{1}^{2}}\right)+\left(\frac{\Delta u_{x}^{j}}{\Delta \omega_{2}^{2}}\right)+\left(\frac{\Delta u_{y}^{j}}{\Delta \omega_{1}^{2}}\right)+\left(\frac{\Delta u_{y}^{j}}{\Delta \omega_{2}^{2}}\right)\right]
$$




$$
\begin{gathered}
\Delta u=u^{i}-u^{d}, \\
\Delta \omega_{1}^{2}=\left(\omega_{1}^{i}\right)^{2}-\left(\omega_{1}^{d}\right)^{2}, \\
\Delta \omega_{2}^{2}=\left(\omega_{2}^{i}\right)^{2}-\left(\omega_{2}^{d}\right)^{2} . \\
F_{2}(z)=\sum_{j=1}^{n_{f}} \frac{(\Delta \varphi)^{2}}{(\Delta \omega)^{2}} \\
\varphi^{2}=\left(\varphi^{i}-\varphi^{d}\right)^{2}=\left(\omega^{i}-\omega^{d}\right)^{2} \\
F_{3}(z)=\frac{\sum_{j=1}^{N}\left|\varphi_{j}^{i} \cdot \varphi_{j}^{d}\right|^{2}}{\left[\sum_{j=1}^{N} \varphi_{j}^{i^{2}}\right] \times\left[\sum_{j=1}^{N} \varphi_{j}^{d^{2}}\right]} \\
F_{4}(z)=\frac{\sum_{j=1}^{N}\left|\omega_{j}^{i} \cdot \omega_{j}^{d}\right|^{2}}{\left[\sum_{j=1}^{N} \omega_{j}^{i^{2}}\right] \times\left[\sum_{j=1}^{N} \omega_{j}^{d^{2}}\right]} \\
\sum_{j=1}^{N}\left(\Delta u_{x}^{j}+\Delta u_{y}^{j}\right) \\
\sum_{j=1}^{K} \Delta \omega_{j}^{2}
\end{gathered}
$$

\section{OTIMIZAÇÃO DAS FUNÇÕES OBJETIVO}

Definidas as assinaturas estruturais resta aplicar a estratégia para a busca dos danos. Para isso define-se uma função resíduo $\mathrm{F}_{\text {res }}^{(\mathrm{j})}(\mathrm{z})$ num espaço métrico adequado (Kolmogorov e Fomin, 1970).

As falhas a serem prognosticadas são apresentados pelo vetor de modelagem do dano, aqui chamado de vetor $\mathrm{z}=\left\{\mathrm{z}_{\mathrm{i}}\right\}=\{\mathrm{b}, \alpha\}$ onde " $\mathrm{b}$ " é o número da barra com o dano, ou seja representa a localização do dano e " $\alpha$ " um número $\alpha \in \mathfrak{R}^{+}$com $\alpha \in(0,1)$, representando a extensão do dano em função da proporção da área da seção transversal da barra.

A função $F_{\text {res }}^{(j)}(z)$ compara a assinatura estrutural escolhida para a avaliação do dano com uma das funções de assinatura estrutural $\mathrm{F}_{\mathrm{j}}\left(\mathrm{z}_{\mathrm{i}}\right)$ entre a situação do dano real $\mathrm{F}_{\mathrm{j}}\left(\mathrm{z}_{\mathrm{r}}\right)$ e de um dano prognosticado $\mathrm{F}_{\mathrm{j}}\left(\mathrm{z}_{\mathrm{i}}\right)$. O vetor $\left\{\mathrm{z}_{\mathrm{i}}\right\}=\left\{\mathrm{b}_{\mathrm{i}}, \alpha\right\}$ indica uma barra $b_{i}$ com fração de seção transversal $\alpha$ prognosticada e $\left\{z_{r}\right\}=\left\{b_{r}, \alpha_{r}\right\}$; sendo $b_{r}$ a barra de localização do dano real e $\alpha_{\mathrm{r}}$ a fração da seção transversal que quantifica o dano real da barra $b_{r}$. $A$ função $F_{r e s}^{(j)}\left(z_{i}\right)$ pode ser matematicamente definida como na Equação 16.

$$
F_{\text {res }}^{(j)}\left(z_{i}\right)=\left\{\left[F_{j}\left(z_{r}\right)-F_{j}\left(z_{i}\right)\right]^{q}\right\}^{1 / q} \quad \text { Eq. [16] }
$$

A constante $q$, segundo (Scales e Gersztenkorn, 1988), define uma métrica que geralmente é usada como norma Euclidiana (i.e. $q=2$ ) muito comumente adotada na minimização de problemas de regressão. Nota-se que o valor de $q=2$ é apropriado para uma hipotética distribuição Gaussiana de erros nos dados de referência $\mathrm{F}_{\mathrm{j}}\left(\mathrm{z}_{\mathrm{r}}\right)$ - para maiores detalhes ver (Scales e Gersztenkorn, 1988) e (Bezerra, 1993).

A localização do dano corresponde ao mínimo da Equação 17, a qual corresponde ao mínimo da Equação 16, isto é:

$$
\operatorname{Min}\left[F_{r e s}^{(j)}\left(z_{i}\right)\right] \rightarrow 0
$$

Quanto mais próximo de zero for o valor da Equação 17, mais próximo o prognóstico $\left\{\mathrm{z}_{\mathrm{i}}\right\}=\left\{\mathrm{b}_{\mathrm{i}}, \alpha\right\}$ estará dos valores medidos reais $\left\{\mathrm{z}_{\mathrm{r}}\right\}=\left\{\mathrm{b}_{\mathrm{r}}, \alpha_{\mathrm{r}}\right\}$, avizinhando-se do dano real. 
Quanto mais distante do zero for o valor da Equação 17, pior o prognóstico; ou seja, mais distante da realidade $\left\{\mathrm{z}_{\mathrm{i}}\right\}=\left\{\mathrm{b}_{\mathrm{i}}, \alpha\right\}$ está de $\left\{\mathrm{z}_{\mathrm{r}}\right\}=\left\{\mathrm{b}_{\mathrm{r}}, \alpha_{\mathrm{r}}\right\}$.

\section{AVALIAÇÃO DAS FUNÇÕES RESÍDUO POR MINIMIZAÇÃO DAS FUNÇÕES OBJETIVO}

A função resíduo $\mathrm{F}_{\text {res }}^{(\mathrm{j})}\left(\mathrm{z}_{\mathrm{i}}\right)$ é expressa em termos de uma assinatura estrutural $\mathrm{F}_{\mathrm{j}}\left(\mathrm{z}_{\mathrm{i}}\right)$. com a finalidade de localizar e quantificar um dano por análise numérico computacional.

Para a análise dos comportamentos das Funções Objetivo com a observação de seus resíduos, é tomada neste trabalho uma estrutura treliçada com 9 barras, sendo utilizado um material com módulo de elasticidade $E=20500 \mathrm{kN} / \mathrm{cm}^{2}$, cargas $\mathrm{Pd}=40 \mathrm{kN}$, como na Figura 3.

As Funções Objetivo são aplicadas na estrutura em que é fixada a barra danificada no diagonal número 6 para todas as funções estudadas. Nesta, serão realizadas duas avaliações, a primeira com um dano real de $20 \%$ da área da seção transversal intacta e a segunda avaliação com um dano real, também no elemento diagonal de número 6, de 20,95\% em relação à área da seção transversal total, correspondendo esse valor a uma redução de $14 \mathrm{~cm} 4$ do momento de inércia do perfil pré-dimensionado para todas as diagonais desta estrutura em análise.

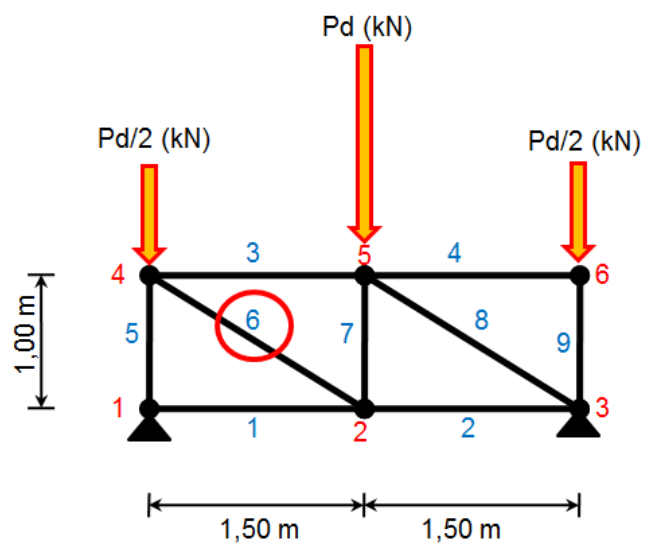

FIGURA 3: Estrutura utilizada para análise das Funções Objetivo, com carregamento e identificação da barra danificada.

FONTE: Próprios autores.
Como nas avaliações dessa estrutura o dano simulado será propagado em $20 \%$, a primeira terá uma resposta exata, já que o dano real proposto foi propositalmente colocado em $20 \%$ da área da seção transversal, de forma que sempre a função estudada no momento terá seus parâmetros prognosticados exatamente igual aos parâmetros simulados, resultando em um mínimo nulo, ao passo que a segunda avaliação possibilitará uma análise comparativa da capacidade das Funções Objetivo em se aproximar com o seu valor mínimo da localização da barra danificada. Nesta segunda avaliação o dano real não será exato $(20,95 \%)$ e sim aproximado isto para que se possa verificar a capacidade da assinatura convergir seu mínimo para a barra danificada e de capturar o dano mesmo não possuindo valores exatos, podendo representar assim possíveis presença de ruídos ou contaminação nos dados coletados.

Busca-se assim, a convergência entre os valores com danos prognosticados, $\mathrm{F}_{\mathrm{j}}\left(\mathrm{z}_{\mathrm{i}}\right)$, que corresponde à aproximação numérica entre $\left\{z_{i}\right\}=$ $\left\{b_{i}, \alpha\right\}$ da estrutura intacta e o valor de referência $\mathrm{F}_{\mathrm{j}}\left(\mathrm{z}_{\mathrm{r}}\right)$ com seu dano real dado por $\left\{\mathrm{z}_{\mathrm{r}}\right\}=\left\{\mathrm{b}_{\mathrm{r}}, \alpha_{\mathrm{r}}\right\}$.

A Figura 4 a seguir mostra uma representação esquemática da utilização do $\mathrm{MMQ}$ na combinação entre as grandezas das funções resíduo utilizadas com a finalidade de localizar na estrutura a barra danificada através da convergência entre os valores admitidos e os valores lidos.

Os comportamentos das funções $F_{j}\left(z_{i}\right)$ são representados a seguir para a estrutura dada na Figura 3 e apresentados seus mínimos na avaliação exata e aproximada, respectivamente.

Na função $F_{1}(z)$, a utilização das principais frequências $\omega_{1}$ e $\omega_{2}$ atingiu seu mínimo na barra danificada, barra 6, apesar de produzir vários pontos próximos de zero, tanto na avaliação exata (20\%) como na aproximada (20,95\%), Figura 5, mostrando-se adequada para o processo de minimização proposto. 


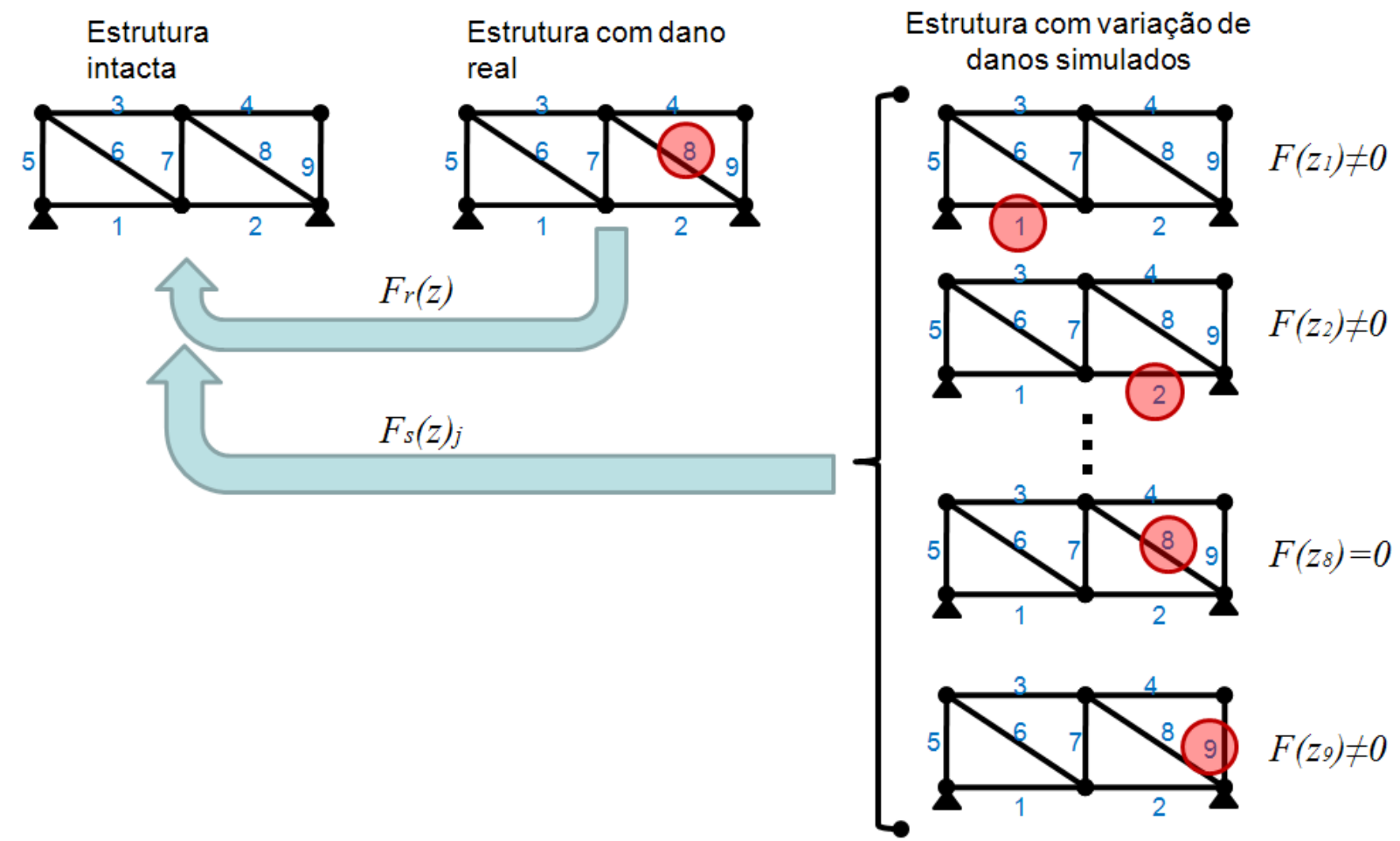

FIGURA 4: Representação esquemática da convergência entre os valores lidos $\operatorname{Fr}(z)$ e os valores simulados ou admitidos $\mathrm{Fs}(\mathrm{z})$.

FONTE: Próprios autores.

A função $F_{2}(z)$, assim como a função $F_{1}(z)$, gera vários pontos de resíduos próximos de zero ao se analisar os elementos da estrutura, tanto na verificação com valor de dano exato $(20 \%)$ como na simulação com dano aproximado (20,95\%). Os pontos encontrados na função $F_{2}(z)$, muito próximos do mínimo, trazem dificuldade práticas em sua leitura, podendo levar a análise a um falso mínimo, não se compatibilizando com a identificação do dano na barra diagonal 6 , podendo este problema ser maximizado ao se analisar estruturas de maior porte, Figura 6.

A função $F_{3}(z)$, denominada COMAC (Coordinate Modal Assurance Criterion), mostra muitos pontos próximos do mínimo, ao contrário do que é obtido na análise aproximada na qual consegue atingir o seu mínimo exatamente na barra onde o dano foi previamente localizado (barra 6), na Figura 7, mostrando ser uma função capaz de atingir mínimos para qualquer dano da estrutura.
A função $F_{4}(z)$, variação da COMAC (Coordinate Modal Assurance Criterion), apresentada anteriormente, mostra um comportamento totalmente oposto ao da função $F_{3}(z)$, na análise exata identifica claramente seu mínimo localizado no elemento de barra danificado bem destacado dos outros elementos da treliça, porém isso não acontece quando realizada a análise de dano aproximado, tendo muitos pontos muito próximos no mínimo não demonstrando confiabilidade em sua utilização na localização de um dano.

A função objeto $F_{5}(z)$, assim como a $F_{1}(z)$ que combina parâmetros estáticos e dinâmicos, destaca com eficiência o ponto mínimo no dano da barra da estrutura proposta, tanto na análise de dano exato como também na realizada com dano aproximado, demonstrando ser uma função objeto de alta confiabilidade no que diz respeito à captura do dano na estrutura. 


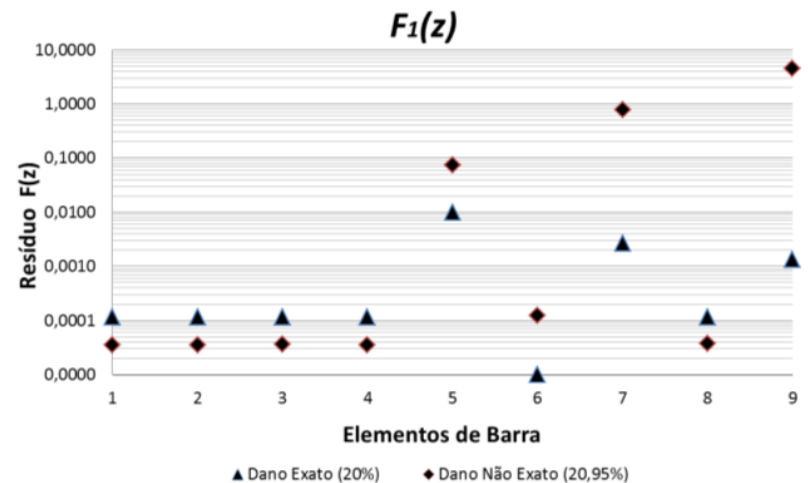

FIGURA 5: Comportamento exato com dano de $20 \%$ e não exato com dano de $20,95 \%$ na Assinatura $F_{1}(z)$ para os elementos da treliça.

FONTE: Próprios autores.

$F_{3}(z)$

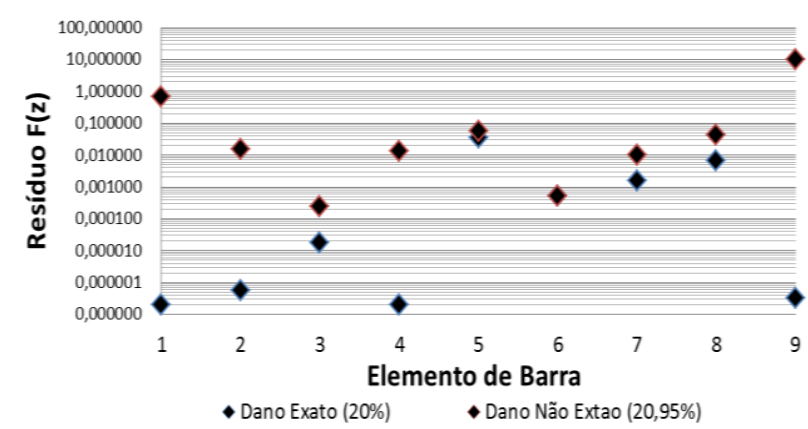

FIGURA 7: Comportamento exato com dano de $20 \%$ e não exato com dano de $20,95 \%$ na Assinatura $F_{3}(z)$ para os elementos da treliça. FONTE: Próprios autores.

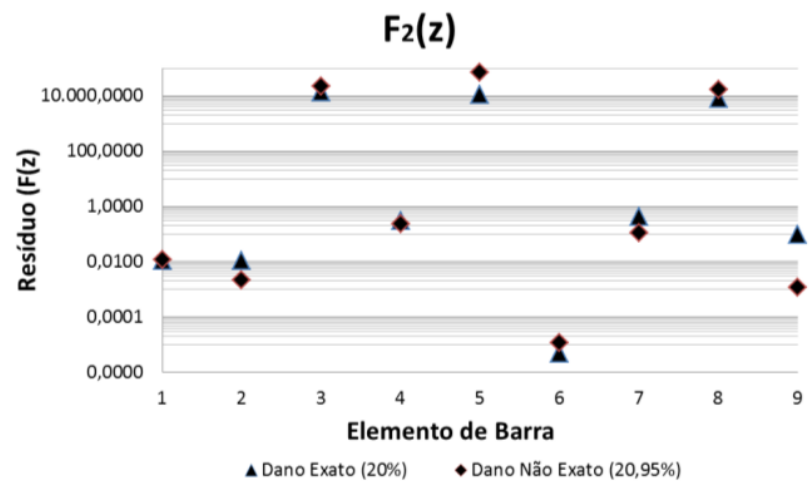

FIGURA 6: Comportamento exato com dano de $20 \%$ e não exato com dano de $20,95 \%$ na Assinatura $F_{2}(z)$ para os elementos da treliça. FONTE: Próprios autores.

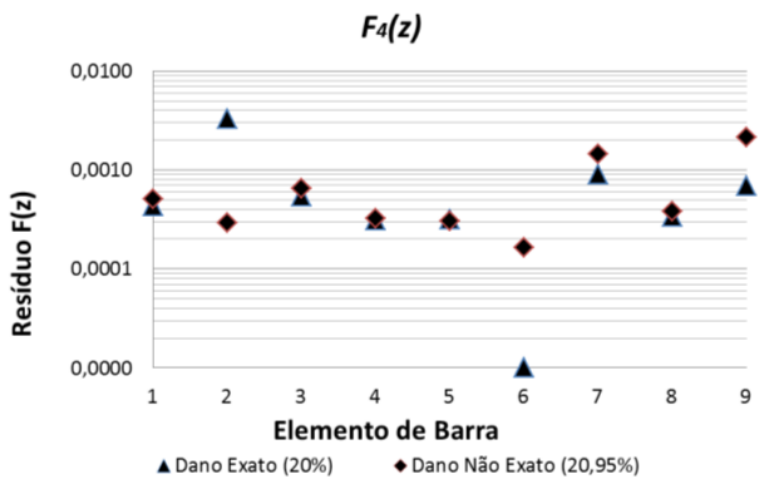

FIGURA 8: Comportamento exato com dano de $20 \%$ e não exato com dano de $20,95 \%$ na Assinatura $F_{4}(z)$ para os elementos da treliça. FONTE: Próprios autores.

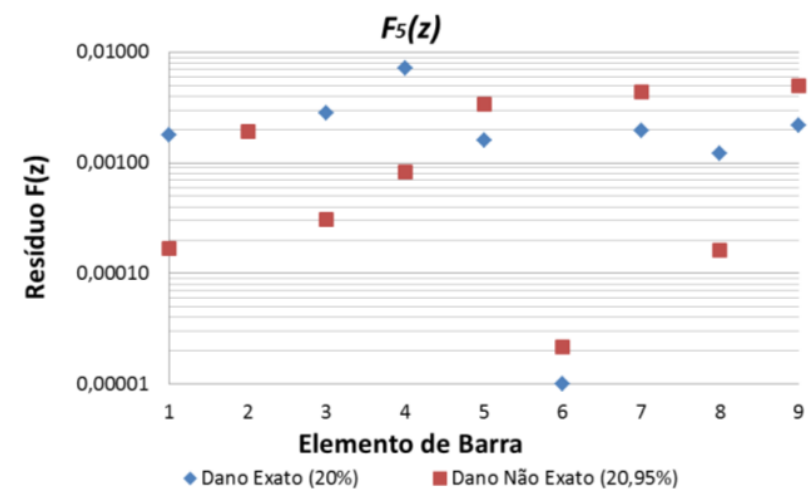

FIGURA 9: Comportamento exato com dano de $20 \%$ e não exato com dano de $20,95 \%$ na Assinatura $F_{5}(z)$ para os elementos da treliça.

FONTE: Próprios autores.

\section{CONCLUSÕES}

Determinar danos em estruturas treliçadas aparenta ser uma tarefa fácil à primeira vista, pois as barras da treliça, a grosso modo, estão sob efeitos apenas de forças axiais. Além disso, um defeito ou vários defeitos numa barra sob esforço axial diminui a capacidade portante desta barra, independentemente da posição ao longo da mesma.
Portanto, encontrar defeito em treliças resume-se em identificar o número da barra $b_{i}$, com i $\in \mathrm{N}^{*}$ e a fração danificada $\alpha$ da seção transversal, com $\alpha \in$ $(0,1)$ que diminui a capacidade portante da barra, ou seja, $\mathrm{b}_{\mathrm{i}}=\mathrm{b}_{\mathrm{r}} \mathrm{e} \alpha \approx \alpha_{\mathrm{r}}$ sendo o índice $\mathrm{r}$ indicativo do dano real. Contudo, o maior problema encontrado na determinação de danos em treliças é a dificuldade da obtenção de assinaturas estruturais 
sensíveis e que gerem funções residuais bemcomportadas e com mínimos bem definidos.

Este trabalho mostra que achar danos em estruturas reticuladas - especificamente treliças e certamente pórticos planos e espaciais não é uma tarefa fácil, tendo em vista que a localização do dano corresponde à minimização da função resíduo que é escrita em termos da assinatura escolhida, a Função Objetivo.

A análise das assinaturas estruturais, tanto as já propostas por alguns autores quanto às propostas inicialmente neste trabalho, foi de fundamental importância para que se houvesse o conhecimento do comportamento quanto às respostas obtidas pelas formulações com grandezas puramente dinâmicas, ou pela combinação entre grandezas estáticas, como por exemplo, os deslocamentos nodais, e grandezas como modos e frequências naturais de vibração da estrutura.

A combinação de parâmetros estáticos, como os deslocamentos nodais, juntamente com os dinâmicos, como as frequências naturais de vibração da estrutura, mostra uma maior eficiência no equacionamento de Funções Objeto destinadas a identificação da localização do dano.

A formulação $F_{5}(z)$ mostra ser dentre as apresentadas, a que possui maior robustez no que diz respeito ao tratamento dos parâmetros utilizados em seu equacionamento, capturando muito bem, tanto na análise exata quanto na análise aproximada, o dano no elemento proposto da treliça estudada.

\section{AGRADECIMENTOS}

Os autores gostariam de agradecer pelo apoio a esta e outras pesquisas à: A Faculdade de Tecnologia da Universidade de Brasília; ao Programa de Pós-Graduação em Estruturas de Construção (PECC); A Faculdade de Engenharia da Universidade Federal de Goiás - Regional Catalão; ao Programa de Pós-Graduação em Modelagem e Otimização (POSMOT) - IMTec; ao Instituo Federal de Brasília IFB; e às Agências de fomento CNPq e CAPES.

\section{REFERÊNCIAS BIBLIOGRÁFICAS}

BEZERRA, L. M. Inverse Elastostatics Solutions with
Boundary Elements. Ph.D. thesis, Carnegie Mellon University, Pittsburgh, PA, 1993.

BEZERRA, L. M.; SAIGAL, S. A Boundary Element Formulation for the Inverse Elastostatics Problem (IESP) of Flaw Detection. International Journal for Numerical Methods in Engineering, USA, v. 36, n. 13, 1993, 2189$2202 \mathrm{p}$.

BRITO, M. A. N. Análise de Assinaturas Elastostáticas e Elastodinâmicas na Solução do Problema Inverso de Identificação de Danos em Treliças Via Método dos Elementos Finitos. Dissertação (Mestrado em Estrutura e Construção Civil), Publicação E.DM-004A/08, Departamento de Engenharia Civil e Ambiental, Universidade de Brasília, Brasília, DF, 2008, 157p.

BRITO, M. A. N.; BEZERRA, L. M. ; SILVA, R.S.Y.C. Analysis of the Structural Integrity of Spectrum through Inverse Response Residual Functions. In: 7th International Conference on Inverse in Engineering, 2011, Orlando, USA. 7 th International Conference on Inverse in Engineering, 2011.

BRITO, M. A. N.; BEZERRA, L. M. ; SILVA, W. T. M. Utilização de Equações Ativas de Restrição em Problemas Inversos Elastostáticos de Identificação de Trincas. In: XXX CILAMCE, 2009, Búzios - RJ. XXX CILAMCE.

BUCHER, H. F.; MAGLUTA, C; MANSUR, W. J. Application of joint time-frequency distribution for estimation of timevarying modal damping ratio. Structural. Engineering and Mechanics, v. 37, 2011, 131-147 p.

GARNIER, C.; PASTOR, M-L.; EYMA, F.; LORRAIN, B. The detection of aeronautical defects in situ on composite structures using Non Destructive Testing. Composite Structures 93, 2011, 1328-1336 p

HALMSHAW, R. Non-Destructive testing. Vitoria, Australia: Edward Arnold, 2011.

KOLMOGOROV A. N., AND S. V. FOMIN. Introdution to real analysis. New York: Dover, 2011.

KUBO, S. Inverse problems related to the mechanics and fracture of solids and structures. JSME Int. Journal. 31, $1988,157-166 \mathrm{p}$.

HU, N.; X. WANG; H. FUKUNAGA; Z.H. YAO; H.X. ZHANG; Z.S. WU. Damage assessment of structures using modal test data. International. Journal of Solids and Structures, Volume 38 , Issue $18,2001,3111-3126 \mathrm{p}$.

OLIVEIRA, M. A. DE. Monitoramento de Integridade Estrutural Baseada em Sensores Piezelétricos e Análise de Sinais no Domínio do Tempo. Tese de Doutorado em Pós-graduação em Engenharia Elétrica. Universidade Estadual Paulista, Campos Ilha Solteira - UNESP, 2013.

SCALES, J. A., and A. GERSZTENKORN. Robust methods in inverse theory. Inverse Problems, 1988, 4: 1071-1091p. 
TANAKA M., AND Y. MASUDA. Boundary element method aplied to some inverse problems. Engineering Analysis, 1986, 3: 138-143 p.

TANAKA M.; M. NAKAMURA, and NAKANO Defect shape identification by means of elastodynamics boundary element analysis and optimization technique. Advances in Boundary Elements. Ed. C. A. Brebbia. Berlin: SpringVerlag, 1988, Vol. 3. 183-194 p.

TOMASZEWSKA, A. Influence of statistical errors on damage detection based on structural flexibility and mode shape curvature. Computers and Structures, 2010, v.88, 154-164 p.

WEN, P.H.; HON, Y.C.; XU, Y. G. Inverse Heat Conduction Problems by Using Particular Solutions. Heat Transfer Asian Research, 2011, 40 (2). 